\title{
Energy-Efficient Train Operation Using Nature-Inspired Algorithms
}

\author{
Kemal Keskin and Abdurrahman Karamancioglu \\ Department of Electrical and Electronics Engineering, Eskisehir Osmangazi University, 26480 Eskisehir, Turkey \\ Correspondence should be addressed to Kemal Keskin; kkeskin@ogu.edu.tr
}

Received 27 July 2016; Revised 5 October 2016; Accepted 25 October 2016; Published 12 January 2017

Academic Editor: Andrea D’Ariano

Copyright (C) 2017 K. Keskin and A. Karamancioglu. This is an open access article distributed under the Creative Commons Attribution License, which permits unrestricted use, distribution, and reproduction in any medium, provided the original work is properly cited.

\begin{abstract}
A train operation optimization by minimizing its traction energy subject to various constraints is carried out using natureinspired evolutionary algorithms. The optimization process results in switching points that initiate cruising and coasting phases of the driving. Due to nonlinear optimization formulation of the problem, nature-inspired evolutionary search methods, Genetic Simulated Annealing, Firefly, and Big Bang-Big Crunch algorithms were employed in this study. As a case study a real-like train and test track from a part of Eskisehir light rail network were modeled. Speed limitations, various track alignments, maximum allowable trip time, and changes in train mass were considered, and punctuality was put into objective function as a penalty factor. Results have shown that all three evolutionary methods generated effective and consistent solutions. However, it has also been shown that each one has different accuracy and convergence characteristics.
\end{abstract}

\section{Introduction}

Researchers have been focusing on new energy saving areas, as energy becomes more expensive besides scarce availability and negative environmental effects in the process of its generation and consumption. One such area is the train operations process where there is a significant potential to reduce energy consumption by optimizing operation strategies. Energy savings through improvements in driving strategies do not require any hardware modification or additional manufacturing costs; therefore, it is the first choice in many cases. In this manuscript we develop energy-efficient driving strategies by choosing switching times among the motion modes of a railway vehicle. In the present context, the motion modes of a railway vehicle are traction, cruising, coasting, and braking modes. Finding the optimal switching times involves solving a nonlinear optimization problem with objective function containing an integral and constraints involving a differential equations set.

In 1968, Ichikawa investigated train operation between successive stations in terms of minimizing energy consumption. A method was proposed to decrease the complexity of the state variable problem and solve it afterwards [1].
In 1975, Hoang et al. tried to reduce energy consumption of train operation from a different viewpoint. They note that positioning of stations and the paths between them are already determined; therefore, remaining task would be determining the "tunnel trace in the equivalent vertical plane." They constructed an optimal control model and provided a heuristic approach using a direct search algorithm. The proposed method was applied to a part of Montreal Subway [2]. There is a line of research where Pontryagin's maximum principle was used to find optimal operation strategy [3-5]. In this line, Howlett showed that an optimal strategy for energy minimization should consist of acceleration, cruising, coasting, and braking phases, respectively [3]. In this respect, illustrative numerical examples of driving strategies for various speeds and gradients took place in $[6,7]$. In 1997, Chang and Sim proposed a coasting control strategy based on genetic algorithm. Punctuality and comfort were taken into account in addition to energy consumption. The proposed method has a strong mathematical basis and it converges in a significantly short time. Also this optimized coast control strategy showed better performance in comparison with fuzzy ATO controller [8]. In another study, Howlett considered the problem using continuous and 
discrete control tools and used the Kuhn-Tucker equations to analyze optimal switching points [9]. Liu and Golovitcher proposed an analytical method based on maximum principle to find optimal input sequence and switching points. Developed system was capable of finding optimal trajectory and suitable for real-time optimization due to its analytical nature [10]. In 2004, Wong and Ho researched to determine number of coasting and switching points by means of three various genetic algorithms [11]. Açikbaş and Söylemez developed simulation software which is suitable for multitrain and multiline tests. They applied artificial neural network and genetic algorithm to model a part of metro line in Istanbul and compared these approaches in terms of energy consumption [12]. In 2009, Howlett et al. presented a new analytical solution for tracks with steep uphill and downhill sections to find optimal switching points [13]. Kim and Chien investigated train operation with constraints of time, speed, and energy consumption. A simulation model was developed [14] and simulated annealing algorithm was utilized to calculate optimal switching points and cruising speed. It was clarified that it was possible to reduce energy consumption by increasing the travel time [15]. In another study, optimization problem was handled by three different methods: dynamic programming, gradient methods, and sequential quadratic programming [16]. In [17], Sheu and Lin focused on automatic train regulation (ATR) problem in the energy efficiency framework. They developed a dual heuristic programming method which brought the ATR ability of real-like adaptation. In addition to controlling coasting points and dwell time at stations, scheduling optimization could increase energy efficiency performance. An energy-efficient driving strategy and optimization method for manually driven high speed trains were developed [18]. Energy-efficient strategies and timetable optimization were combined together in [18-22]. For subway systems, recovered energy coming from regenerative braking can be used for train traction. In this regard, Yang et al. developed scheduling rules that synchronized successive trains for braking and accelerating. Overlapping time was maximized by means of integer programming model and for timetable optimization genetic algorithm was utilized [23]. Lu et al. investigated energy-efficient train trajectory by means of dynamic speed control. Ant colony optimization, genetic algorithm, and dynamic programming algorithms were used in searching the optimal trajectory [24]. Su et al. proposed a new approach by combining optimal driving strategy with cooperative train control. The energy which came from regenerative braking is used for traction of other trains [25]. Similarly, while one research controlled headway time and dwell time to increase energy savings from regenerative braking [26] the other one developed stop-skipping method [27] to decrease passenger waiting time. In another research, a concept of dynamic infrastructure occupation was presented to assess infrastructure capacity under disturbed conditions as a complement to the established capacity indicator of scheduled infrastructure occupation. This new indicator is applied in a capacity assessment study of a Dutch railway corridor with different signaling configurations under both scheduled and disturbed traffic conditions [28]. During the recent years, several other methods were applied to optimal train control problem such as fuzzy predictive control [29], BellmanFord algorithm [30], reinforcement learning [31], swarm optimization [32], and NSGA-II algorithm [33].

In this study an energy-efficient train operation problem was considered on a track with no steep sections. Three heuristic approaches, Firefly Algorithm, Genetic Simulated Annealing, and Big Bang-Big Crunch, were used to find switching points for phases. There is no known study on train operation optimization problem which employs one of these algorithms. In this study, it was demonstrated that these three algorithms are appropriate to apply to the energyefficient train operation optimization problem and a comparison between algorithms running times and optimality was discussed. Besides the fact that the train model and the track were real-like modeled, the effect of number of passengers on train energy consumption and algorithm's performance were evaluated. However, the problem in case study was solved successfully; for a complicated track with steep sections or speed limitations more complex strategies are required (see $[5,7,9,10,13])$.

In the next section we present the nonlinear optimization formulation of the problem. In Section 3, the evolutionary solution methods used in this manuscript are introduced. In the 4 th section, the solution methods are applied to a model of locally existing real problem. In the remaining part of the manuscript performances of the methods are discussed.

\section{Modeling the Motion}

The motion equations of train, using Newton's second law, can be written in the following form:

$$
\begin{aligned}
& \frac{d x}{d t}=v, \\
& \frac{d v}{d t}=\frac{F_{t}-F_{b}}{m}-R-R_{g},
\end{aligned}
$$

where $x$ and $v$ are position and speed of the train, respectively. $F_{t}$ is the tractive force, $F_{b}$ is the braking force, $R$ is the rolling resistance, $R_{g}$ is the resistance caused by level change, and $m$ denotes mass of train. In the sequel, a train motion on a sequence of successive stations is considered. We denote the distance between stations $i$ and $i+1$ by $X_{i}$, allowed travel time by $T_{i}$, and allowed maximum speed by $V$. Hence, between stations $i$ and $i+1$, these parameters are restricted with following limits:

$$
\begin{aligned}
& 0 \leq x \leq X_{i}, \\
& 0 \leq t \leq T_{i}, \\
& 0 \leq v \leq V
\end{aligned}
$$

Resistance of the train, $R$, can be calculated by utilizing Davis equation [34]:

$$
R=A+B v+C v^{2}
$$

where the coefficients $A, B$ and $C$ correspond to mass, mechanical, and air resistance, respectively. These coefficients 


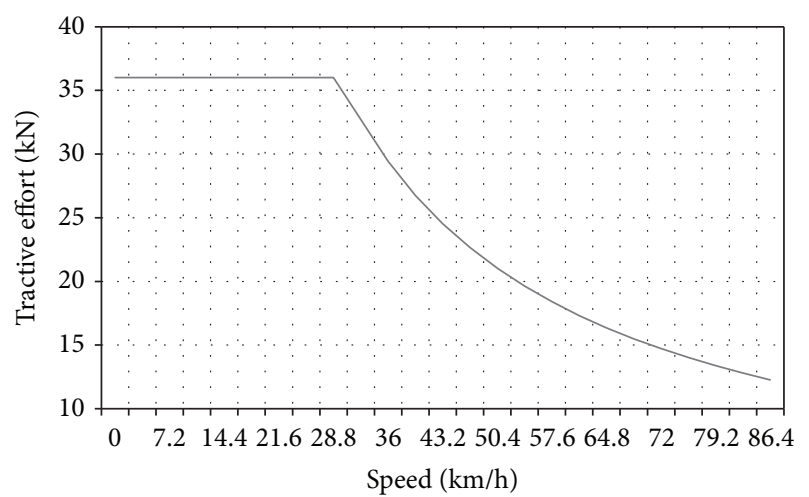

FIGURE 1: Tractive effort, speed graph for a tram with power $571 \mathrm{hp}$.

vary depending on external forces and physical characteristics of train. Level changes in track can be favorable even though they can function as a resistance against to the train motion. For downhill part of track the contribution to the acceleration is positive and for uphill part of track, it is negative. The resistance caused by gradient can be calculated as follows:

$$
R_{g}=g \sin \alpha,
$$

where $g$ is gravitational acceleration and $\alpha$ is the angle of slope. Tractive effort provides force to move train along the rail line.

Tractive effort is specifically defined according to train's characteristics. It is restricted to certain limits due to adhesion between wheel and rail surfaces. Tractive effort calculation mostly depends on engine power and current speed of train (see Figure 1). Maximum tractive effort is available at low speed. For the beginning of motion maximum tractive effort (here it is $36 \mathrm{kN}$ ) is applied until train reaches $30 \mathrm{~km} / \mathrm{h}$ speed. Over this speed level, power takes its maximum constant value and tractive effort changes inverse proportional to speed. It can be calculated by [35]

$$
F_{t}=2650 \frac{\mu P}{v},
$$

where $\mu$ is the efficiency in converting motor power to tractive force, $P$ is motor power, $v$ is the current speed of train, and 2650 is for unit conversion. Using this equation average power can be calculated:

$$
P=\frac{F_{t} v}{2650 \mu}
$$

The total energy consumption is obtained by integrating the power over time:

$$
E=\int_{0}^{T} P d t
$$

2.1. Train Operation. Energy consumption of a train considerably depends on train operation. An optimal train operation for a level track should consist of the following

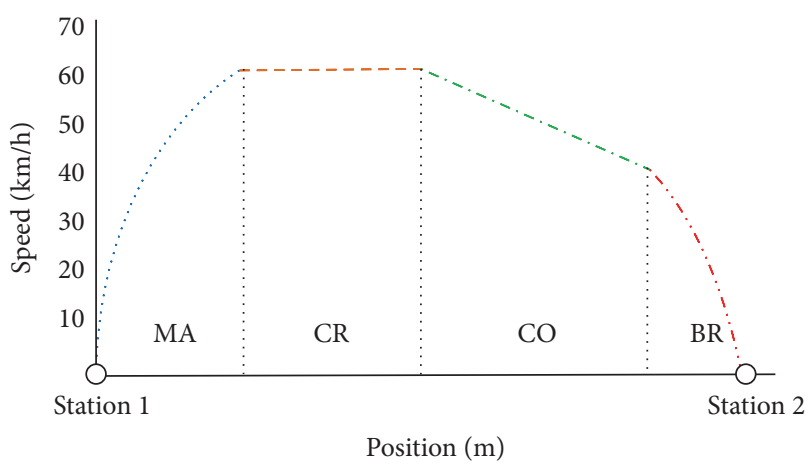

FIGURE 2: Motion phases: MA: maximum acceleration, CR: cruising, $\mathrm{CO}$ : coasting, and BR: braking.

motion phases, respectively: maximum acceleration (MA), cruising (CR), coasting (CO), and braking (BR) [3]. An example driving scenario between two successive stations is shown in Figure 2. Next we provide more details on the motion phases below.

2.1.1. Maximum Acceleration (MA). From beginning of the travel till the start of the cruising phase, maximum acceleration is applied to the train. As mentioned earlier, tractive effort is restricted with adhesion limit. The maximum tractive effort is calculated by

$$
F_{\max }=m g \eta,
$$

where $m$ is the mass of train, $g$ is gravitational acceleration, and $\eta$ is the friction constant. Under an applied constant power, tractive effort stays constant until train comes to the cruising speed value.

2.1.2. Cruising (CR). In this phase train continues its travel at a constant speed. In order to hold the speed at constant value, the applied tractive effort must equal the opposing forces to the train motion. Uphill and downhill sections of track either contribute to or take away from the amount of tractive effort. For some downhill sections, there may be no need for traction.

2.1.3. Coasting (CO). In the coasting phase, train moves along a line under already obtained momentum and no traction energy is consumed. This phase continues until train reaches the safe stopping distance. Safe stopping distance is a function of remaining distance and current speed of train. The safe stopping distance is

$$
x_{\mathrm{ss}}=\frac{v_{\mathrm{br}}^{2}}{2 a}
$$

where $v_{\mathrm{br}}$ is current speed subject to braking, $a$ is deceleration, and $x_{\mathrm{ss}}$ is safe stopping distance. On tracks with nonsteep constant gradient, optimal braking speed which depends only on the uniquely defined cruising speed can be calculated by (9). This formula is efficient for level tracks. 
2.1.4. Braking $(B R)$. In this phase, constant force opposing the direction of motion is applied to train. Magnitude of braking force depends on train characteristics. Since we just consider traction power for calculation of energy consumption, in braking phase, it is assumed that there is no contribution to total energy consumption.

2.2. Total Energy Consumption and Optimization. Total energy consumption for an optimal strategy can be decomposed into its motion phases. In the maximum acceleration phase the traction effort is fixed at its attainable maximum value; therefore, speed monotonically increases. The endpoint of this phase is denoted by $k_{1}$. In the cruising phase, speed is fixed to a value, $v_{\mathrm{cr}}$, which requires $F_{t}$ values to equal the opposing resistance values. This phase extends between the points $k_{1}$ and $k_{2}$. The points $k_{1}$ and $k_{2}$ are called the switching points. In the subsequent phases (i.e., coasting and braking phases), no energy is consumed due to zero traction force. Thus, total energy consumption for an optimal strategy throughout successive stations is calculated by the following equation:

$$
E_{\mathrm{TOTAL}}=E_{\mathrm{MA}}+E_{\mathrm{CR}}
$$

where $E_{\mathrm{MA}}$ and $E_{\mathrm{CR}}$ denote energies consumed at the maximum acceleration and cruising phases. Equation (10) is valid for track with nonsteep sections. It is desired to provide energy-efficient travel while considering punctuality and comfort. A correct decision-making on the switching points between the phases has primary significance for the problem under consideration since it determines the energy consumption. Finding optimal switching points for a travel between stations $i$ and $i+1$ can be formulated as an optimization problem:

$$
\begin{array}{cl}
\min _{k_{1}, k_{2}} & \left(\int_{0}^{k_{1}} F_{t}(t) v(t) d t+\int_{k_{1}}^{k_{2}} F_{t}(t) v_{c r} d t\right) \\
\text { Subject to: } & \frac{d v(t)}{d t}=\frac{F_{t}(t)-F_{b}(t)}{m}-R(t)-R_{g}(t), \\
& \frac{d x(t)}{d t}=v(t), \quad 0 \leq t \leq T_{i}, \\
& F_{t}(t)=F_{t \max }, \quad 0 \leq t \leq T_{i}, \\
& F_{t}(t) \\
=5+0.0285 v_{\mathrm{cr}}+0.0047 v_{\mathrm{cr}}^{2}+m g \alpha, & \\
F_{t}(t)=0, \quad k_{2}<t \leq T_{i}, \\
F_{b}(t)=0, \quad 0<t \leq k_{3},
\end{array}
$$

$$
\begin{aligned}
& F_{b}(t)=F_{b \max }, \quad k_{3}<t \leq T_{i}, \\
& 0 \leq F_{t} \leq F_{t \max }, \\
& 0 \leq F_{b} \leq F_{b \max }, \\
& v(0)=v\left(T_{i}\right)=0, \\
& 0 \leq v(t) \leq v\left(T_{i}\right) \\
& x(0)=0, \\
& x\left(T_{i}\right)=X_{i} .
\end{aligned}
$$

Having formulated the energy optimization problem above, in the next section, for its solution, we present a review of three different evolutionary algorithms.

\section{Optimization Methods}

In this section, Genetic Simulated Annealing, Firefly, and Big Bang-Big Crunch algorithms are reviewed briefly, where the former one is a hybrid algorithm and the latter two are standalone algorithms.

3.1. Genetic Simulated Annealing Algorithm. Genetic Algorithm (GA) and Simulated Annealing (SA) are two wellknown tools for solving global optimization problems. GA is an evolutionary search method based on evolutionary theory. Search procedure mimics the natural genetics using operators such as selection, mutation, and crossover. Chromosomes refer to candidate solutions and each of them is assigned a score with regard to fitness function. New offspring are generated by applying genetic operators to chromosomes. After several generations, chromosomes which have better scores are selected as optimal or suboptimal solution. SA is another nature-inspired optimization method which shows an analogy to physical annealing process in metallurgy. In the physical process temperature is reduced gradually in the cooling phase of the heated material in order to prevent defects. In the mathematical counterpart, SA starts to search from an initial point and next new candidate solutions are generated randomly by reducing temperature. From new generations, not only better solutions but also some worse solutions are accepted with a certain probability. Thus, local minima can be avoided and the chance of finding optimum solution is increased [36]. The algorithms GA and SA have stand-alone features which can be used together to eliminate each one's typical weaknesses. GA employs the efficiency of evolution theory such that new offspring have several characteristics in common with its parent. In this way, the quality of solutions is maintained. With the help of its extensive search capability, GA is practical for solving tough problems. However, besides the uncertainty of computational time, it can be incapable of avoiding local extrema in limited time as well [37]. With the help of random search nature, SA accepts worse solutions in addition to better ones with a certain rate. It prevents being caught to a local extremum [38]. 


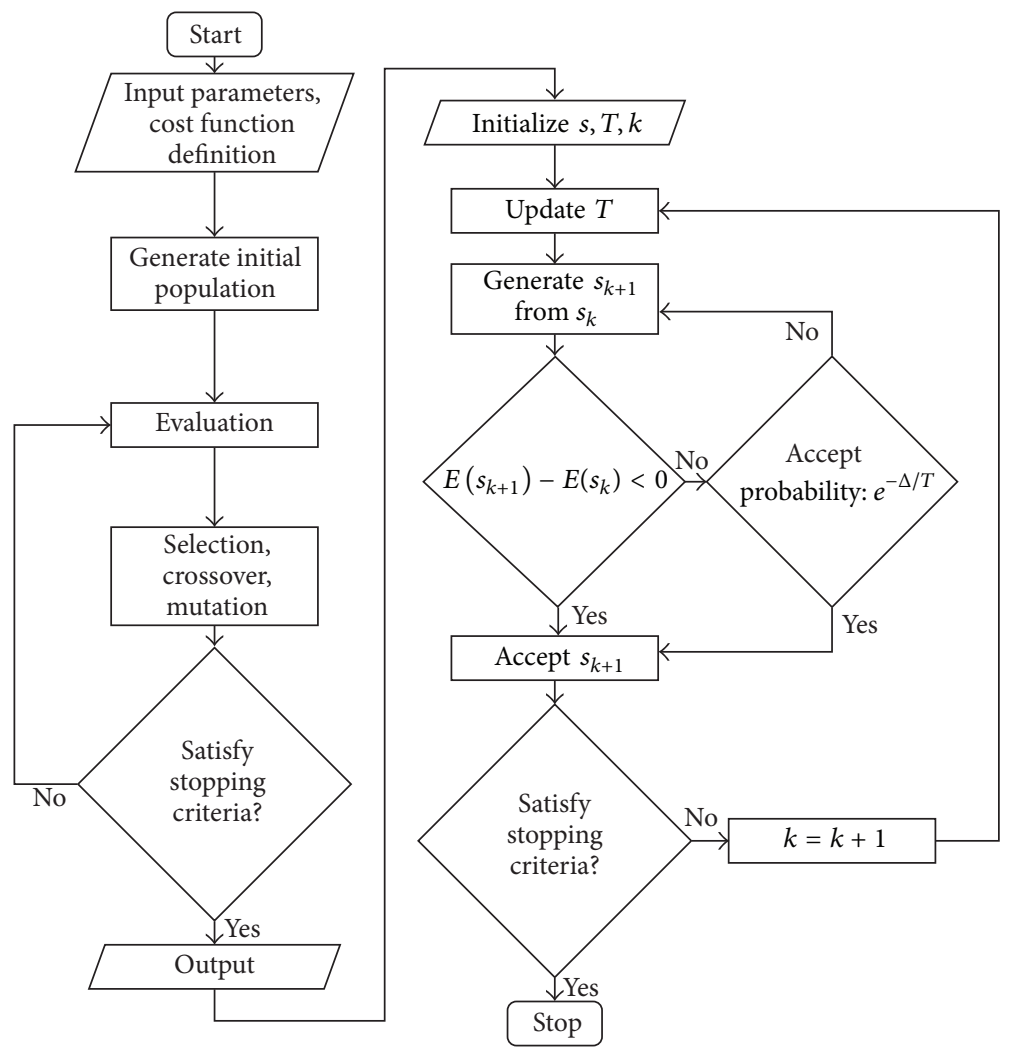

Figure 3: Flowchart of Genetic Simulated Annealing algorithm.

Even though the SA can avoid local extrema, its efficiency depends on initial point. Choosing inappropriate initial point may result in worse solutions and a long computational time.

Genetic Simulated Annealing (GSA) is a combination of GA and SA. At the beginning of algorithm, initialization parameters such as population size, number of variables, lower and upper bounds for each variable, mutation and crossover rates, selection method, annealing, and temperature functions are defined. Then GA part of algorithm is activated and stopping criteria are defined as a certain number of generations. At the end of this part of algorithm a suboptimal solution is generated. The second part of algorithm employs SA with the initial solution from the first part. Algorithm flowchart is given in Figure 3.

GSA has been applied to many areas including job scheduling [39], multiple project scheduling [40], discrete time-cost tradeoff [41], traveling salesman and error correcting code design [42], mixed-model assembly line sequencing [43], and train energy optimization [36] problems.

3.2. Big Bang-Big Crunch Method. Big Bang-Big Crunch (BB$\mathrm{BC})$ is a global optimization method which is inspired by the formation of the universe. BB-BC method comprises two main phases: big bang and big crunch. At the big bang phase, individuals from initial population scatter along the search space randomly. On that sense, this phase of algorithm has resemblance to GA. After random initialization of population, individuals take various places in search space.
Random number generators are adjusted to certain values to hold new offspring in the search space. Then big crunch phase follows the big bang phase. An output point, namely, center of mass, is generated based on population data. This crunch process can be formulated for a minimization problem as [44]

$$
s^{c}=\frac{\sum_{i=1}^{K}\left(1 / f^{i}\right) s^{i}}{\sum_{i=1}^{K}\left(1 / f^{i}\right)}
$$

where $s^{c}$ is the center of mass, $s^{i}$ is the position vector for the $i$ th individual, $f^{i}$ represents the fitness value of the $i$ th individual, and $K$ is the population size. After the big crunch phase it is required to create new members which will be used in next iteration of big bang phase. New population is generated around the center of mass using following formulation:

$$
s_{i}^{\text {new }}=s^{c}+\sigma,
$$

where $s_{i}^{\text {new }}$ stands for new population's $i$ th individual and $\sigma$ is standard deviation coefficient. Through (13), new individuals cannot go out of search space. Standard deviation coefficient is calculated by

$$
\sigma=\frac{0.5 r\left(s_{\max }-s_{\min }\right)}{1+j / h},
$$

where $r$ is a random number which is defined with normal distribution; $j$ is iteration number; $s_{\max }$ and $s_{\min }$ are the upper 
and lower limits for search space, respectively; $h$ is coefficient for the contract of search space. For subsequent iterations the center of mass is calculated again and big bang, big crunch steps are repeated until a stopping criterion is met. Algorithm steps can be given as follows:

(1) Create a random initial population with $K$ members.

(2) Calculate the fitness function of every individual.

(3) Calculate center of mass using (12).

(4) Create new candidates by using (13).

(5) Return to step (2) until stopping criteria have been met.

Although BB-BC algorithm has been announced in recent years, it has been applied many areas including design of space trusses [45], nonlinear controller design [46], fuzzy model inversion [47], damage detection [48], and energyefficient motion control of train [49] problems.

3.3. Firefly Algorithm. Firefly Algorithm (FA) is a swarm intelligence method inspired by lightning behavior of fireflies. It was proposed by Yang in 2008 [50]. FA mainly depends on three significant ideas:

(i) Fireflies have no gender. Any of them can be attracted to other fireflies.

(ii) Attractiveness is comparative to brightness. For instance, considering two flashing fireflies, one which has less glitter will move towards to more glitter one. When distance increases, attractiveness and brightness decrease expectedly. If both fireflies are not glittery enough to attract other one, then random movement occurs.

(iii) The view of objective function defines the brightness of a firefly. It is possible to express brightness in different ways; however, a basic one may make use of the objective function of the relevant maximization problem.

Two issues are worth attention for firefly algorithm: light intensity and attractiveness. Essentially, the light intensity $I(d)$ can be defined using the inverse square law [50]:

$$
I(d)=\frac{I_{s}}{d^{2}},
$$

where $I_{s}$ refers to the intensity at source and $d$ is the distance between fireflies. Attractiveness is directly related to the light intensity seen by neighbor fireflies. Let $\beta$ be attractiveness of a firefly; it can be defined as

$$
\beta=\beta_{0} e^{\gamma d^{2}},
$$

where $\beta_{0}$ denotes the attractiveness at $d=0$ and $\gamma$ is light absorption coefficient. The distance between two fireflies $i$ and $j$ at points $p_{i}$ and $p_{j}$ can be defined as follows [50]:

$$
d_{i j}=\left\|p_{i}-p_{j}\right\|=\sqrt{\sum_{k=1}^{l}\left(p_{i, k}-p_{j, k}\right)^{2}},
$$

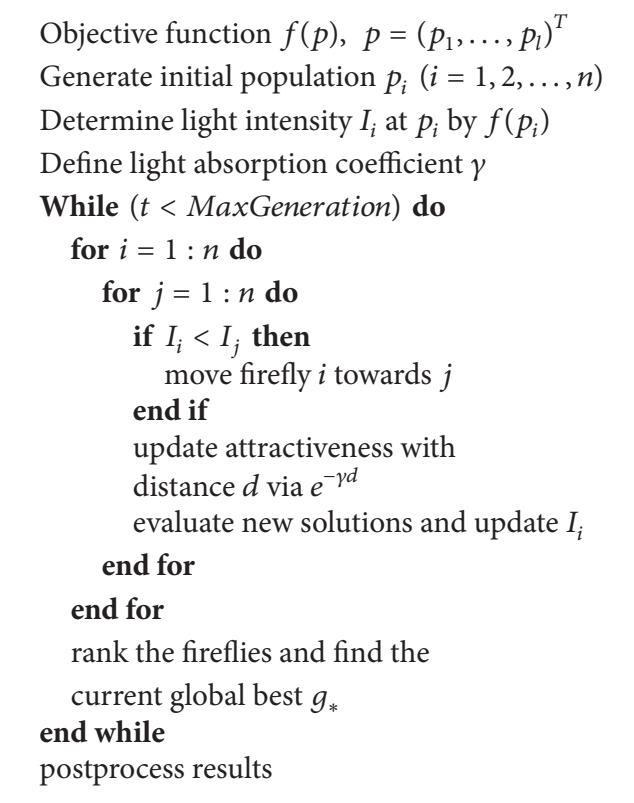

Algorithm 1: Firefly algorithm [50].

where $p_{i, k}$ is the $k$ th component of the spatial coordinate $p_{i}$ of $i$ th firefly. $\|\cdot\|$ denotes the Euclidean norm, and $l$ denotes the number of components. Also the movement of firefly $i$ to firefly $j$ is determined by

$$
p_{i}=p_{i}+\beta_{0} e^{-\gamma d_{i j}^{2}}\left(p_{j}-p_{i}\right)+\alpha \varepsilon_{i},
$$

where second term refers to attraction and the third term represents randomization, and $\alpha$ is randomization parameter. Regarding to the information given above, algorithm's pseudo code is shown in Algorithm 1.

FA has been applied to many areas including learning robot motion trajectories [51], heart disease prediction [52], and arterial cannula shape optimization [53] problems.

\section{A Case Study}

This research focuses on energy optimization for an urban rail transit system. In this regard, different searching methods for global optimization problem have been described in the previous sections. In order to verify the efficiency of proposed optimization algorithms, a case study and its results for each method are given in this section.

4.1. Case Study Background. A particular segment of Eskisehir Urban Rail Network was taken into account for the case study and a real-like tram model was created with characteristics which are given in Table 1.

The total length of test track is $3314 \mathrm{~m}$. There are seven stations where the train must stop (see Figure 4). Travel starts at Osmangazi University station and ends at Stadyum station. Considering successive stations, train motion can be examined in partial tracks. To interpret the figure as 


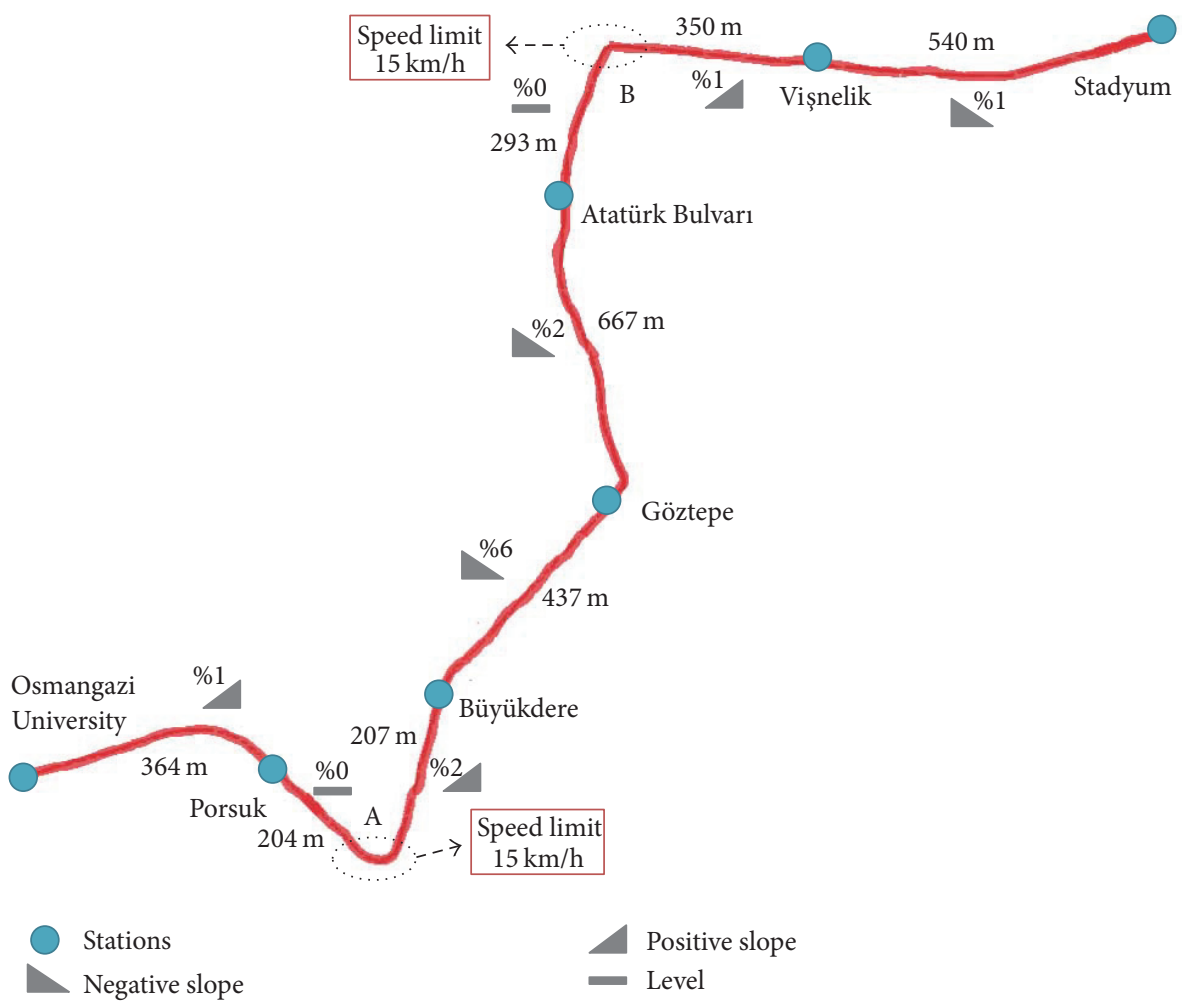

FIgURE 4: A part of Eskisehir light rail network subjected to test.

TABLE 1: Train characteristics.

\begin{tabular}{lc}
\hline Total mass & $34000 \mathrm{~kg}$ \\
Maximum motor power & $571 \mathrm{hp}$ \\
Number of cars & $5 \mathrm{pcs}$ \\
Max speed limit & $70 \mathrm{~km} / \mathrm{h}$ \\
Capacity & 150 passengers \\
\hline
\end{tabular}

intended, let us read the figure for the first three stations. At the beginning, train starts its motion from Osmangazi University station and stops at Porsuk station. The length of this part is $364 \mathrm{~m}$ and there is $1 \%$ positive grade. The second part of total track is between Porsuk station and the following first sharp curvature. This part is $204 \mathrm{~m}$ long with no gradient. Train speed goes down to $15 \mathrm{~km} / \mathrm{h}$ at the end of this part and keeps it at this level along the curvature. After passing the curvature, new part begins between the curvature and Buyukdere station. Since the train comes from previous part with $15 \mathrm{~km} / \mathrm{h}$ constant speed, it starts to accelerate from $15 \mathrm{~km} / \mathrm{h}$ in this part. This part's length is $207 \mathrm{~m}$ and has $2 \%$ positive slope.

4.2. Operation Strategy. Only the MA and CR phases contribute to the energy consumption of the train. As no energy is consumed in $\mathrm{CO}$ phase, increasing duration of $\mathrm{CO}$ phase in a strategy leads to drop in energy consumption. However, this affects the total travel time adversely. Energy efficiency
TABLE 2: Estimated motion phases for the parts of track.

\begin{tabular}{lcc}
\hline Part of Track & Length & $\begin{array}{c}\text { Estimated Phase } \\
\text { Sequence }\end{array}$ \\
\hline $\begin{array}{l}\text { Osmangazi University - } \\
\text { Porsuk }\end{array}$ & $364 \mathrm{~m}$ & $\mathrm{MA}+\mathrm{CR}+\mathrm{CO}+\mathrm{BR}$ \\
Porsuk - Curvature A & $204 \mathrm{~m}$ & $\mathrm{MA}+\mathrm{CR}+\mathrm{BR}$ \\
Curvature A - Buyukdere & $207 \mathrm{~m}$ & $\mathrm{MA}+\mathrm{CR}+\mathrm{BR}$ \\
Buyukdere - Goztepe & $437 \mathrm{~m}$ & $\mathrm{MA}+\mathrm{CR}+\mathrm{CO}+\mathrm{BR}$ \\
Goztepe - Ataturk Bulvari & $667 \mathrm{~m}$ & $\mathrm{MA}+\mathrm{CR}+\mathrm{CO}+\mathrm{BR}$ \\
Ataturk Bulvar1 - & $293 \mathrm{~m}$ & $\mathrm{MA}+\mathrm{CR}+\mathrm{BR}$ \\
Curvature B & $350 \mathrm{~m}$ & $\mathrm{MA}+\mathrm{CR}+\mathrm{CO}+\mathrm{BR}$ \\
Curvature B - Visnelik & $540 \mathrm{~m}$ & $\mathrm{MA}+\mathrm{CR}+\mathrm{CO}+\mathrm{BR}$ \\
Visnelik - Stadyum & &
\end{tabular}

should be provided by adhering to punctuality. Therefore, punctuality takes place in the optimization scheme as a hard constraint, and no tradeoff is allowed between punctuality and energy consumption.

An optimum trajectory for short distances does not consist of CO phase [3]. In this study, the parts with under $350 \mathrm{~m}$ length is considered as a short distance. Regarding this, a predicted motion phase sequence for each part of track is given in Table 2. Thus, the search algorithms to be employed use this granted motion phase sequences, and this contributes efficiency of the search processes. 
TABLE 3: GSA parameter selection test.

\begin{tabular}{|c|c|c|c|c|c|c|c|}
\hline Test label & Crossover rate & Mutation rate & $\begin{array}{l}\text { Selection } \\
\text { function }\end{array}$ & $\begin{array}{l}\text { Crossover } \\
\text { function }\end{array}$ & $\begin{array}{c}\text { Annealing } \\
\text { function }\end{array}$ & $\begin{array}{c}\text { Temperature } \\
\text { function }\end{array}$ & Energy cons. \\
\hline GSA 1 & 0.8 & 0.01 & Roulette & Single-point & Boltzmann & Boltzmann & $5.10 \mathrm{kwh}$ \\
\hline GSA 2 & 0.9 & 0.02 & Tournament & Two-point & Boltzmann & Boltzmann & $5.19 \mathrm{kwh}$ \\
\hline GSA 3 & 0.7 & 0.04 & Roulette & Intermediate & Boltzmann & Boltzmann & $5.18 \mathrm{kwh}$ \\
\hline GSA 4 & 0.8 & 0.01 & Roulette & Single-point & Fast & Exponential & $5.12 \mathrm{kwh}$ \\
\hline GSA 5 & 0.9 & 0.02 & Tournament & Two-point & Fast & Exponential & $5.14 \mathrm{kwh}$ \\
\hline GSA 6 & 0.7 & 0.04 & Roulette & Intermediate & Fast & Exponential & $5.15 \mathrm{kwh}$ \\
\hline
\end{tabular}

4.3. Optimization Parameters. In train operation research area, optimization of speed profile of a train has a challenging mathematical structure. It is desired to find switching points for certain motion phases to minimize energy consumption by taking constraints on physical limitations, time, and comfort into consideration. It is important to note that switching motion phases from one to another is an NP-hard problem [54]. Since analytical approaches have limitations in finding a solution to this problem, evolutionary methods become prominent instead [15].

For the train model under consideration, to test the evolutionary optimization methods, a simulator was developed in MATLAB environment. It takes variable track alignments, speed, and comfort limitations into consideration. In this setup, output consists of speed, position, and time values and energy consumption of train.

In this research, Genetic Simulated Annealing, Firefly, and Big Bang-Big Crunch algorithms were separately employed to minimize energy consumption of a train. Performances of the methods rely significantly on their parameter settings. The chosen parameters for each method are presented below.

4.3.1. Genetic Simulated Annealing Parameters. This method is a combination of two well-known algorithms. The first one, Genetic algorithm (GA), is capable of finding suboptimal solutions in short computational times. Herewith, at the beginning of optimization, GA was employed until it reaches a fitting generation. Obtained solution was given to the second algorithm, simulated annealing algorithm (SA), as an initial solution. For the GA part, it is significant to determine not only crossover and mutation rates but also selection and crossover functions, whereas temperature and annealing function are important parameters for second part of the method.

For satisfactory results GSA needs to have well-chosen parameter settings. These settings are generally selected by repeated trial and error. To reduce the computational burden in this process, a simplified test track, in our case $2000 \mathrm{~m}$ single track with various gradients and no curvature, is used. In the parameter setting process, the costs obtained for various conditions are given in Table 3 . Noting that the test labeled GSA 1 has the best cost, we use its settings for the actual problem with the test track shown in Figure 4. A brief summary of the settings is as follows: (i) population size: 75 ,

(ii) crossover rate: 0.8 ,

(iii) mutation rate: 0.01 ,

(iv) selection function: roulette,

(v) crossover function: single point,

(vi) annealing function: Boltzmann.

4.3.2. Big Bang-Big Crunch Algorithm Parameters. For Big Bang-Big Crunch algorithm, finding new solution candidates is achieved by adding a random number to the center of mass. This random number value is chosen to be decreased as iteration number increases. Parameters which belong to Big Bang-Big Crunch algorithm are given as follows:

(i) population size: 75 ,

(ii) initial point: for each variable to be optimized, average of its attainable minimum and maximum values,

(iii) random number: $r_{k+1}=r_{k} \cdot 10^{-4 / N}$ where $k$ and $N$ are the iteration and generation numbers.

4.3.3. Firefly Algorithm Parameters. Attractiveness and light absorption coefficient are two significant parameters to determine the speed of convergence and efficiency of firefly algorithm. For the simulations to be carried out, the algorithm parameters were heuristically chosen as follows:

(i) population size: 75 ,

(ii) attractiveness, $\beta: 0.2$,

(iii) light absorption coefficient, $\gamma: 1$,

(iv) randomization number, $\alpha: 0.5$.

4.4. Simulation Results. In the case study we apply GSA, FA, and BB-BC algorithms to solve the train speed trajectory optimization problem. To display the performance robustness of the algorithms, for the test track in Figure 4, the simulations were performed for three different total travel times: 345 secs; 350 secs, and 360 secs. Furthermore, for the same purpose, two cases (with no passenger and with passengers) were taken into account. 
TABle 4: Energy consumption (kwh) for different time limits (no passenger).

\begin{tabular}{lccc}
\hline Total travel time & FA & GSA & BB-BC \\
\hline $345 \mathrm{~s}$ & 10.45 & 10.18 & 9.85 \\
$350 \mathrm{~s}$ & 10.26 & 10.00 & 9.78 \\
$360 \mathrm{~s}$ & 10.16 & 9.84 & 9.39 \\
\hline
\end{tabular}

4.4.1. Case I. In this case, where the train has no passenger, train starts its motion from Osmangazi University station and travel ends at Stadyum station (see Figure 4). There are five more stations between departure and arrival stations. Train should stop at each of these stations. For the sake of simplicity in presentation, dwell times are disregarded. The alteration of gradient through the test track is given numerically in Figure 4 and graphically in Figure 5(a). There are two sharp curvatures on track where train speed needs to be limited. At these points train speed is constrained to $15 \mathrm{~km} / \mathrm{h}$. Speed limits for the test track is shown on the speedposition graphics in Figure 5(b).

Simulations using GSA, FA, and BB-BC algorithms were conducted with the parameters given in the previous subsection. Optimization results for total travel time of 350 secs are given in the form of speed trajectories in Figure 6.

Interpreting the optimal speed trajectories in Figure 6, it is noticed that, between the first two stations, all the algorithms result in all the motion phases. However, between the 2nd and 3rd stations, BB-BC and FA algorithms result in no coasting phase and give only the MA, CR, and BR phases. For this part, the GSA proposes only the phases MA and BR. A similar distinctive outcome by the GSA algorithm also occurs between Ataturk Bulvari and Visnelik stations where it eliminates CR phase and apply only the MA, CO, and BR phases. For the other parts, the sequence of motion phases complies with those shown in Table 2. Operation strategy is controlled by determining speed levels for each phase. Maximum speeds of BB-BC, GSA, and FA solutions are $56 \mathrm{~km} / \mathrm{h}, 63 \mathrm{~km} / \mathrm{h}$, and $55 \mathrm{~km} / \mathrm{h}$, respectively. The simulations for Case I are conducted for three different total travel time limits, and for each algorithm corresponding energy costs are shown in Table 4.

Regarding the costs illustrated in Table 4, for every total travel time limit, $\mathrm{BB}-\mathrm{BC}$ demonstrates superior performance compared to GSA and FA solutions. When BB$\mathrm{BC}$ is employed, energy consumption is reduced by $6 \%$ and $3.34 \%$ compared to FA and GSA, respectively. Thus, it can be concluded that BB-BC has better cost performance compared to the other two methods.

4.4.2. Case II. Train mass is a major factor affecting the energy consumption adversely. In this case, optimal driving strategies are searched for the train loaded with varying number of passengers. In this case, certain number of passengers is assumed to get in the train at every station in order to evaluate the impact of passenger load. An exemplary number of passengers just before train departs the indicated station are given in Table 5. Assuming the average mass of an adult
TABLE 5: Number of passengers at each station.

\begin{tabular}{lc}
\hline Station & Number of passengers \\
\hline Osmangazi University & 0 \\
Porsuk & 17 \\
Buyukdere & 41 \\
Goztepe & 54 \\
Ataturk Bulvari & 97 \\
Visnelik & 114 \\
\hline
\end{tabular}

TABLE 6: Energy consumption (kwh) for different time limits (with passenger).

\begin{tabular}{lccc}
\hline Total travel time & FA & GSA & BB-BC \\
\hline $345 \mathrm{~s}$ & 11.52 & 11.28 & 10.95 \\
$350 \mathrm{~s}$ & 11.34 & 10.95 & 10.62 \\
$360 \mathrm{~s}$ & 10.69 & 10.35 & 10.02 \\
\hline
\end{tabular}

TABLE 7: Average convergence results.

\begin{tabular}{lccc}
\hline & FA & GSA & BB-BC \\
\hline Convergence (generation) & 24 & 56 & 44 \\
\hline
\end{tabular}

passenger is $86 \mathrm{~kg}$ [55], train's mass at the stations is shown graphically in Figure 7.

Apart from the train's mass, keeping Case I conditions intact, the speed trajectory corresponding to 350 secs total travel time is given in Figure 8.

Regarding Figure 6 a likewise interpretation of Figure 8 is possible. Energy consumption corresponding to three different total travel times is shown in Table 6.

The $\mathrm{BB}-\mathrm{BC}$, as in the previous case, exhibits a better performance compared to the other two. When BB-BC is employed, energy consumption is reduced by $5.84 \%$ and $3 \%$ on average compared to FA and GSA, respectively. Although there is an increment in train mass approximately by $28 \%$, energy consumption increases by $11 \%$. The results show that the GSA and FA algorithms perform reasonably well under the conditions where the train mass changes throughout the simulation. However, the results also show that these two algorithms are outperformed by the BB-BC algorithm.

4.5. Discussion. Even though the heuristic optimization methods have common features, they differ in each other not only in terminology but also in algorithmic structure. All three methods are evolving population based methods where each member of a population is a solution candidate. Randomness is significant for global optimization tools in terms of exploring new solutions along the search space. With the advantage of being a hybrid algorithm, GSA employs both GA and SA to satisfy randomness. FA attributes randomness to firefly's motion whereas BB-BC provides it as energy dissipation.

The results in Tables 4 and 6 were in terms of optimal costs. Table 7 illustrates convergence rate features of the algorithms. 
(a)

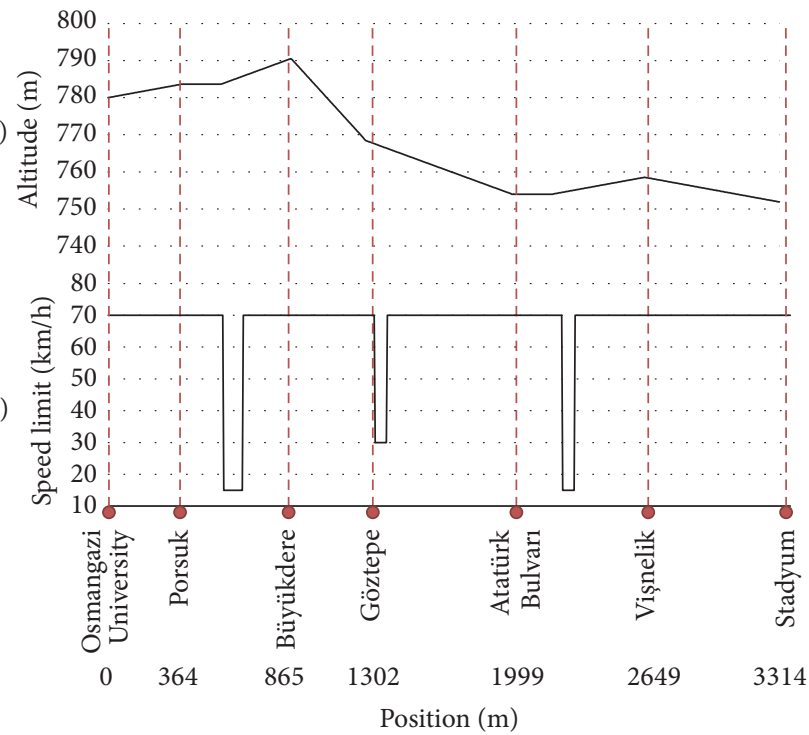

FIgURE 5: Altitude (a) and speed limitation (b).

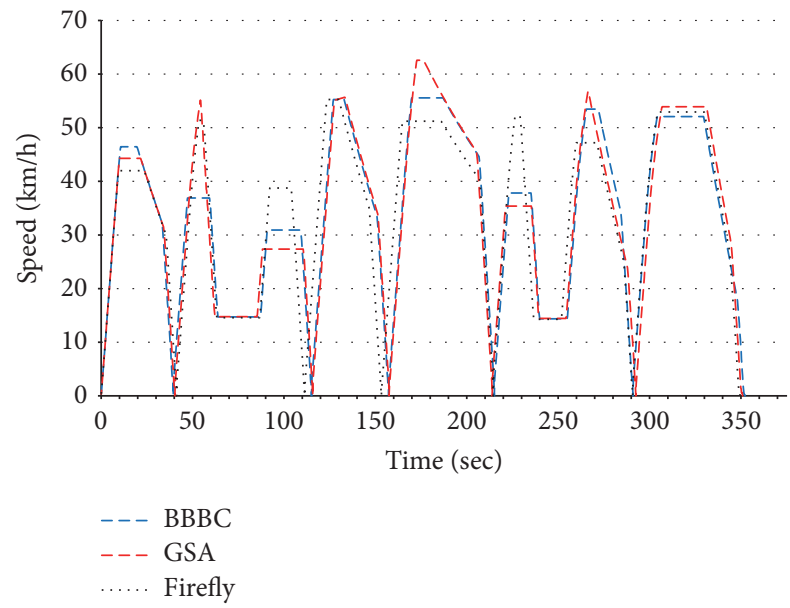

FIGURE 6: Speed-time graph for all algorithms (no passenger).

From what we can observe from Table 7, FA converged to a solution faster than the others. However, its provided solution is mediocre compared to the others. For the optimizations which have restrictions or have time problems caused by slow simulation model and infrastructure, FA algorithm might provide practical solutions. In spite of slow convergence rate, $\mathrm{BB}-\mathrm{BC}$ generates the lowest energy consumption. Therefore, for the optimizations which need more efficient solution and have appropriate simulation environment, BB-BC might be employed. GSA provides better solutions compared to FA but it suffers from convergence.

\section{Conclusion}

In this manuscript, optimal train operation strategies are developed using three nature-inspired metaheuristic algorithms Genetic Simulated Annealing, Firefly, and Big BangBig Crunch. Their performances are tested via MATLAB

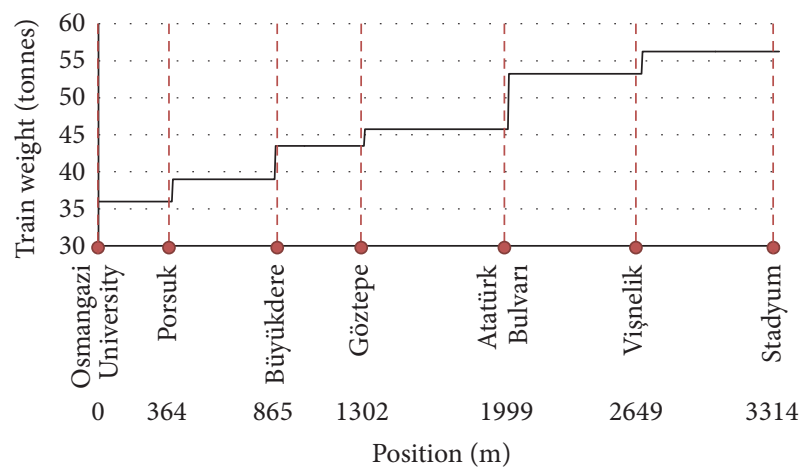

FIgURE 7: Train mass for each station.

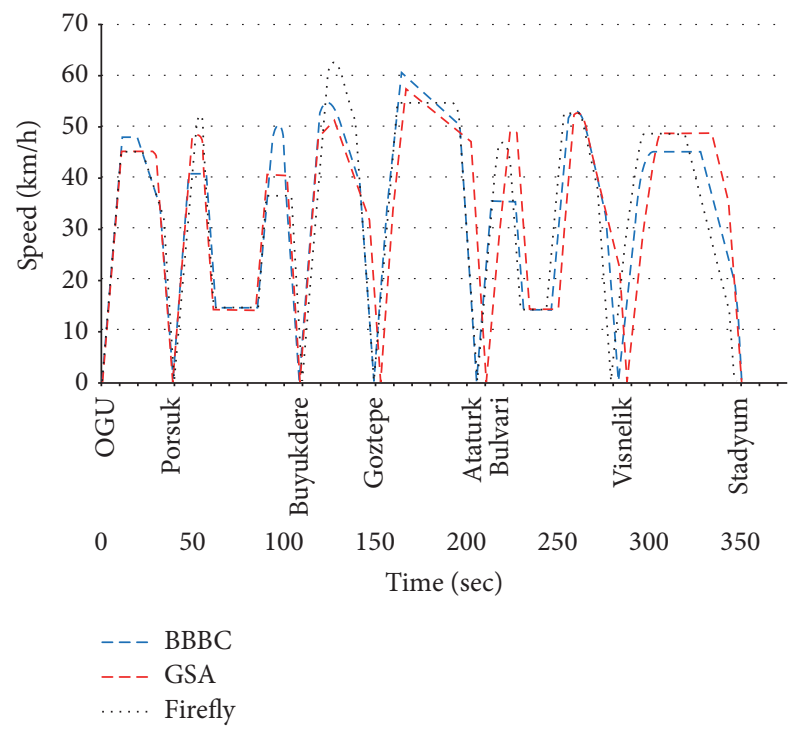

FIGURE 8: Speed-time graph for all algorithms (with passenger). 
simulations for a local rail line under various test conditions. The simulations take track alignments, speed limitations, and train mass into consideration. GSA, FA, and BB-BC searching methods were compared for finding the optimal speed trajectory. Besides various track alignments and speed limitations, changes in train mass are also considered to provide real-like model.

Obtained results may be summarized as follows: when chosen appropriate parameters, GSA method is influential at providing solutions close to the optimal ones. Although FA converges to the solution in short times, it still performs mediocre solutions. All algorithms give consistent results for both no passenger and with passenger conditions. While $\mathrm{BB}-\mathrm{BC}$ reaches the lowest cost solution, it takes a significant computational time. The main contribution of this manuscript is the illustration of successful applicability of three metaheuristic optimization methods to the optimal train operation problem.

\section{Competing Interests}

The authors declare that they have no competing interests.

\section{Acknowledgments}

This work was supported in part by the Eskisehir Osmangazi University Scientific Research Foundation under Grant no. 2015-772.

\section{References}

[1] K. Ichikawa, "Application of optimization theory for bounded state variable problems to the operation of train," Bulletin of JSME, vol. 11, no. 47, pp. 857-865, 1968.

[2] H. H. Hoang, M. P. Polis, and A. Haurie, "Reducing energy consumption through trajectory optimization for a metro network," IEEE Transactions on Automatic Control, vol. 20, no. 5, pp. 590-595, 1975.

[3] P. Howlett, "An optimal strategy for the control of a train," Australian Mathematical Society. Journal. Series B. Applied Mathematics, vol. 31, no. 4, pp. 454-471, 1990.

[4] I. P. Milroy, Aspect of automatic train control [Ph.D. dissertation], Loughborough University of Technology, Sydney, Australia, 1980.

[5] E. Khmelnitsky, "On an optimal control problem of train operation," IEEE Transactions on Automatic Control, vol. 45, no. 7, pp. 1257-1266, 2000.

[6] C. Jiaxin and P. Howlett, "Application of critical velocities to the minimisation of fuel consumption in the control of trains," Automatica, vol. 28, no. 1, pp. 165-169, 1992.

[7] P. Howlett, "Optimal strategies for the control of a train," Automatica, vol. 32, no. 4, pp. 519-532, 1996.

[8] C. S. Chang and S. S. Sim, "Optimising train movements through coast control using genetic algorithms," IEE Proceedings-Electric Power Applications, vol. 144, no. 1, p. 65, 1997.

[9] P. Howlett, "The optimal control of a train," Annals of Operations Research, vol. 98, pp. 65-87, 2000.
[10] R. Liu and I. M. Golovitcher, "Energy-efficient operation of rail vehicles," Transportation Research Part A: Policy and Practice, vol. 37, no. 10, pp. 917-932, 2003.

[11] K. K. Wong and T. K. Ho, "Dynamic coast control of train movement with genetic algorithm," International Journal of Systems Science, vol. 35, no. 13-14, pp. 835-846, 2004.

[12] S. Açikbaş and M. T. Söylemez, "Coasting point optimisation for mass rail transit lines using artificial neural networks and genetic algorithms," IET Electric Power Applications, vol. 2, no. 3, pp. 172-182, 2008.

[13] P. G. Howlett, P. J. Pudney, and X. Vu, "Local energy minimization in optimal train control," Automatica, vol. 45, no. 11, pp. 2692-2698, 2009.

[14] K. Kim and S. I.-J. Chien, "Simulation-based analysis of train controls under various track alignments," Journal of Transportation Engineering, vol. 136, no. 11, pp. 937-948, 2010.

[15] K. Kim and S. I.-J. Chien, "Optimal train operation for minimum energy consumption considering track alignment, speed limit, and schedule adherence," Journal of Transportation Engineering, vol. 137, no. 9, pp. 665-674, 2011.

[16] M. Miyatake and H. Ko, "Optimization of train speed profile for minimum energy consumption," IEEJ Transactions on Electrical and Electronic Engineering, vol. 5, no. 3, pp. 263-269, 2010.

[17] J.-W. Sheu and W.-S. Lin, "Energy-saving automatic train regulation using dual heuristic programming," IEEE Transactions on Vehicular Technology, vol. 61, no. 4, pp. 1503-1514, 2012.

[18] A. P. Cucala, A. Fernández, C. Sicre, and M. Domínguez, "Fuzzy optimal schedule of high speed train operation to minimize energy consumption with uncertain delays and driver's behavioral response," Engineering Applications of Artificial Intelligence, vol. 25, no. 8, pp. 1548-1557, 2012.

[19] S. Su, X. Li, T. Tang, and Z. Gao, "A subway train timetable optimization approach based on energy-efficient operation strategy," IEEE Transactions on Intelligent Transportation Systems, vol. 14, no. 2, pp. 883-893, 2013.

[20] C. Sicre, A. P. Cucala, A. Fernández, and P. Lukaszewicz, "Modeling and optimizing energy-efficient manual driving on high-speed lines," IEEJ Transactions on Electrical and Electronic Engineering, vol. 7, no. 6, pp. 633-640, 2012.

[21] L. Yang, K. Li, Z. Gao, and X. Li, "Optimizing trains movement on a railway network," Omega, vol. 40, no. 5, pp. 619-633, 2012.

[22] S. Su, T. Tang, and C. Roberts, "A cooperative train control model for energy saving," IEEE Transactions on Intelligent Transportation Systems, vol. 16, no. 2, pp. 622-631, 2015.

[23] X. Yang, X. Li, Z. Gao, H. Wang, and T. Tang, "A cooperative scheduling model for timetable optimization in subway systems," IEEE Transactions on Intelligent Transportation Systems, vol. 14, no. 1, pp. 438-447, 2013.

[24] S. Lu, S. Hillmansen, T. K. Ho, and C. Roberts, "Singletrain trajectory optimization," IEEE Transactions on Intelligent Transportation Systems, vol. 14, no. 2, pp. 743-750, 2013.

[25] S. Su, T. Tang, X. Li, and Z. Gao, "Optimization of multitrain operations in a subway system," IEEE Transactions on Intelligent Transportation Systems, vol. 15, no. 2, pp. 673-684, 2014.

[26] X. Yang, B. Ning, X. Li, and T. Tang, "A two-objective timetable optimization model in subway systems," IEEE Transactions on Intelligent Transportation Systems, vol. 15, no. 5, pp. 1913-1921, 2014.

[27] Y. Wang, B. De Schutter, T. J. J. van den Boom, B. Ning, and T. Tang, "Efficient Bilevel approach for urban rail transit operation with stop-skipping," IEEE Transactions on Intelligent Transportation Systems, vol. 15, no. 6, pp. 2658-2670, 2014. 
[28] R. M. P. Goverde, F. Corman, and A. D’Ariano, "Railway line capacity consumption of different railway signalling systems under scheduled and disturbed conditions," Journal of Rail Transport Planning \& Management, vol. 3, no. 3, pp. 78-94, 2013.

[29] Y. Bai, T. K. Ho, B. Mao, Y. Ding, and S. Chen, "Energyefficient locomotive operation for Chinese mainline railways by fuzzy predictive control," IEEE Transactions on Intelligent Transportation Systems, vol. 15, no. 3, pp. 938-948, 2014.

[30] S. Lu, P. Weston, S. Hillmansen, H. B. Gooi, and C. Roberts, "Increasing the regenerative braking energy for railway vehicles," IEEE Transactions on Intelligent Transportation Systems, vol. 15, no. 6, pp. 2506-2515, 2014.

[31] J. Yin, D. Chen, and L. Li, "Intelligent train operation algorithms for subway by expert system and reinforcement learning," IEEE Transactions on Intelligent Transportation Systems, vol. 15, no. 6, pp. 2561-2571, 2014.

[32] M. Domínguez, A. Fernández-Cardador, A. P. Cucala, T. Gonsalves, and A. Fernández, "Multi objective particle swarm optimization algorithm for the design of efficient ATO speed profiles in metro lines," Engineering Applications of Artificial Intelligence, vol. 29, pp. 43-53, 2014.

[33] W. Carvajal-Carreño, A. P. Cucala, and A. Fernández-Cardador, "Optimal design of energy-efficient ATO CBTC driving for metro lines based on NSGA-II with fuzzy parameters," Engineering Applications of Artificial Intelligence, vol. 36, pp. 164-177, 2014.

[34] T. Davis, Transportation Energy Data Book, Center for Transportation Analysis, Washington, DC, USA, 20th edition, 2000.

[35] W. W. Hay, Railroad Engineering, John Wiley \& Sons, New York, NY, USA, 2nd edition, 1982.

[36] K. Keskin and A. Karamancioglu, "A hybrid optimization algorithm for energy efficient train operation," in Proceedings of the International Symposium on Innovations in Intelligent SysTems and Applications (INISTA '15), pp. 1-6, Madrid, Spain, September 2015.

[37] A. Chen, T. Jiang, Z. Chen, and Y. Zhang, "A genetic and simulated annealing combined algorithm for optimization of wideband antenna matching networks," International Journal of Antennas and Propagation, vol. 2012, Article ID 251624, 6 pages, 2012.

[38] J. Wang, Q. Duan, Y. Jiang, and X. Zhu, "A new algorithm for grid independent task schedule: genetic simulated annealing," in Proceedings of the 2010 World Automation Congress (WAC '10), pp. 165-171, Kobe, Japan, September 2010.

[39] L. Wang and D. Zheng, "An effective hybrid optimization strategy for job-shop scheduling problems," Computers \& Operations Research, vol. 28, no. 6, pp. 585-596, 2001.

[40] P.-H. Chen and S. M. Shahandashti, "Hybrid of genetic algorithm and simulated annealing for multiple project scheduling with multiple resource constraints," Automation in Construction, vol. 18, no. 4, pp. 434-443, 2009.

[41] R. Sonmez and Ö. H. Bettemir, "A hybrid genetic algorithm for the discrete time-cost trade-off problem," Expert Systems with Applications, vol. 39, no. 13, pp. 11428-11434, 2012.

[42] H. Chen, N. S. Flann, and D. W. Watson, "Parallel genetic simulated annealing: a massively parallel SIMD algorithm," IEEE Transactions on Parallel and Distributed Systems, vol. 9, no. 2, pp. 126-136, 1998.

[43] M. Rabbani, S. Sadri, N. Manavizadeh, and H. Rafiei, "A novel bi-level hierarchy towards available-to-promise in mixedmodel assembly line sequencing problems," Engineering Optimization, vol. 47, no. 7, pp. 947-962, 2015.
[44] O. K. Erol and I. Eksin, "A new optimization method: Big Bang-Big Crunch," Advances in Engineering Software, vol. 37, no. 2, pp. 106-111, 2006.

[45] C. V. Camp, "Design of space trusses using big bang-big crunch optimization," Journal of Structural Engineering, vol. 133, no. 7, pp. 999-1008, 2007.

[46] M. Doğan and Y. Istefanopulos, "Optimal nonlinear controller design for flexible robot manipulators with adaptive internal model," IET Control Theory \& Applications, vol. 1, no. 3, pp. 770778, 2007.

[47] T. Kumbasar, İ. Eksin, M. Güzelkaya, E. Yesil, İ. Eksin, and E. Yeşil, "Big bang big crunch optimization method based fuzzy model inversion," in MICAI 2008: Advances in Artificial Intelligence: 7th Mexican International Conference on Artificial Intelligence, Atizapán de Zaragoza, Mexico, October 27-31, 2008 Proceedings, vol. 5317 of Lecture Notes in Computer Science, pp. 732-740, Springer, Berlin, Germany, 2008.

[48] Z. Tabrizian, E. Afshari, G. G. Amiri, M. H. Ali Beigy, and S. M. P. Nejad, "A new damage detection method: big Bang-Big Crunch (BB-BC) algorithm," Shock and Vibration, vol. 20, no. 4, pp. 633-648, 2013.

[49] K. Keskin and A. Karamancioglu, "Energy efficient motion control for a light rail vehicle using the big bang big crunch algorithm," in Proceedings of the 14th IFAC Symposium on Control in Transportation Systems (CTS '16), pp. 442-446, Istanbul, Turkey, May 2016.

[50] X. S. Yang, Nature-Inspired Metaheuristic Algorithms, Luniver Press, London, UK, 2008.

[51] M. Mitić and Z. Miljković, "Bio-inspired approach to learning robot motion trajectories and visual control commands," Expert Systems with Applications, vol. 42, no. 5, pp. 2624-2637, 2015.

[52] N. C. Long, P. Meesad, and H. Unger, "A highly accurate firefly based algorithm for heart disease prediction," Expert Systems with Applications, vol. 42, no. 21, pp. 8221-8231, 2015.

[53] K. Tesch and K. Kaczorowska, "Arterial cannula shape optimization by means of the rotational firefly algorithm," Engineering Optimization, vol. 48, no. 3, pp. 497-518, 2016.

[54] V. Xuan, Analysis of Necessary Conditions for the Optimal Control of a Train, University of South Australia, 2006.

[55] Federal Aviation Administration, Aircraft Weight and Balance Control, Flights Standards Service, Washington, DC, USA, 2005. 


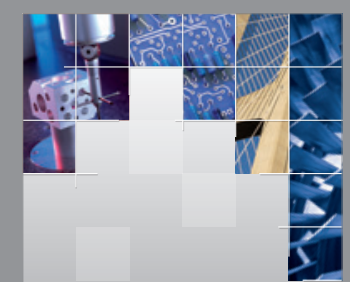

\section{Enfincering}
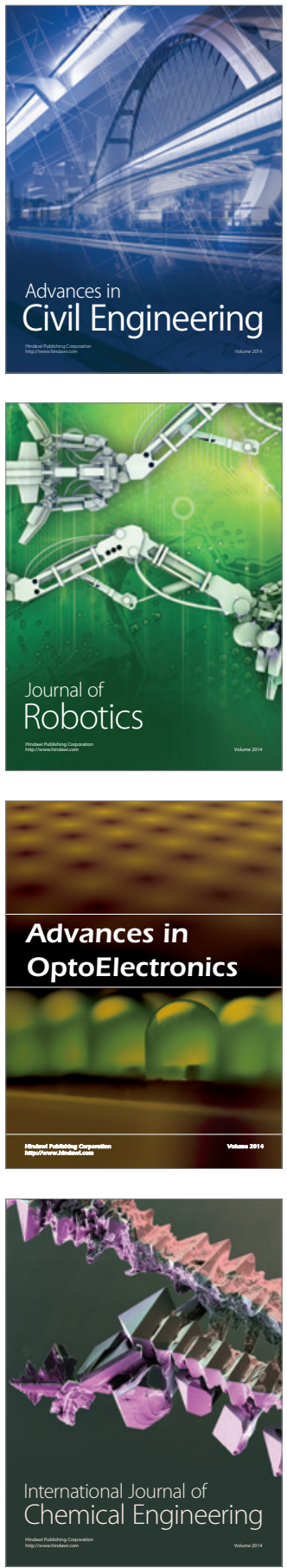

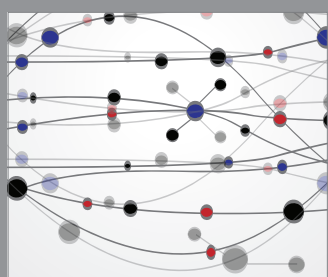

The Scientific World Journal

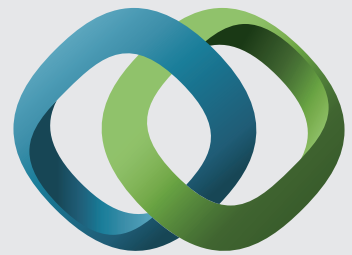

\section{Hindawi}

Submit your manuscripts at

https://www.hindawi.com
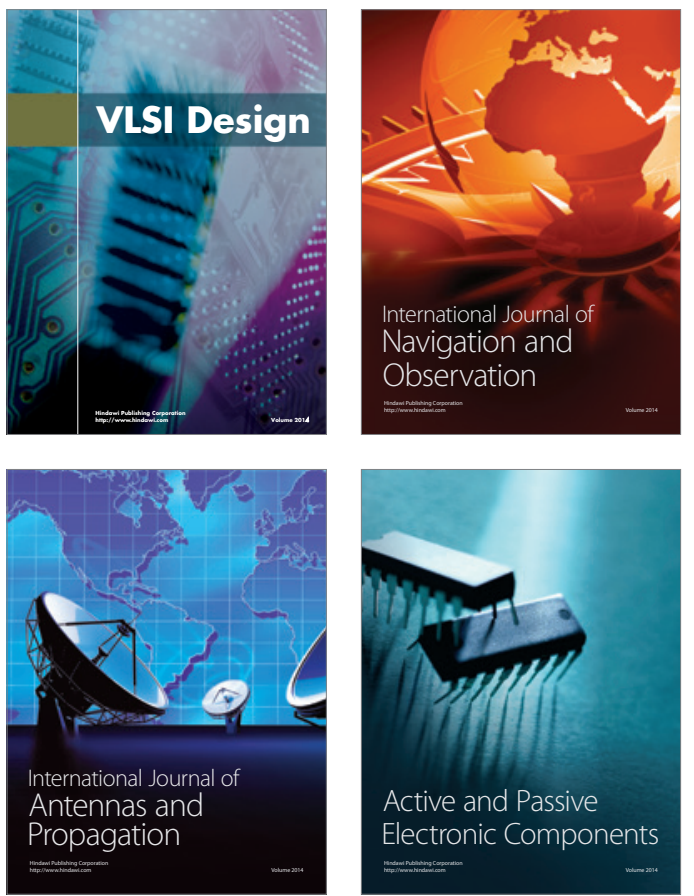
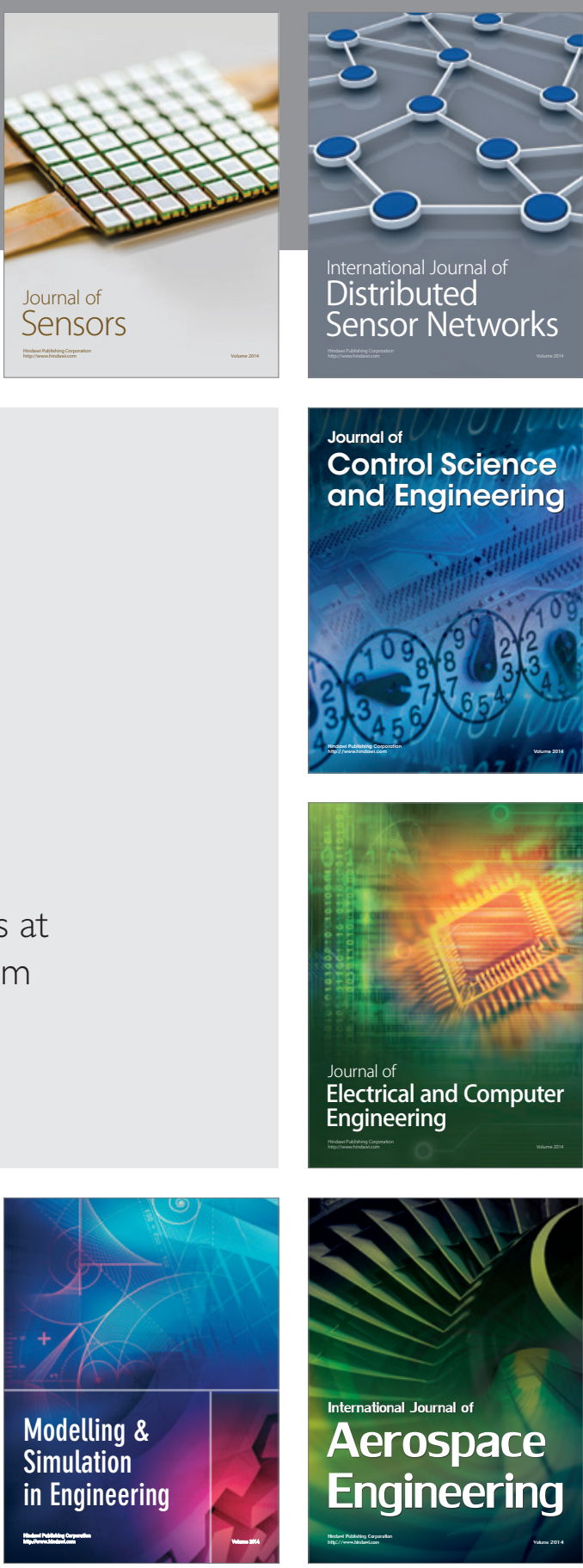

International Journal of

Distributed

Sensor Networks

$-$

Joumal of

Control Science

and Engineering
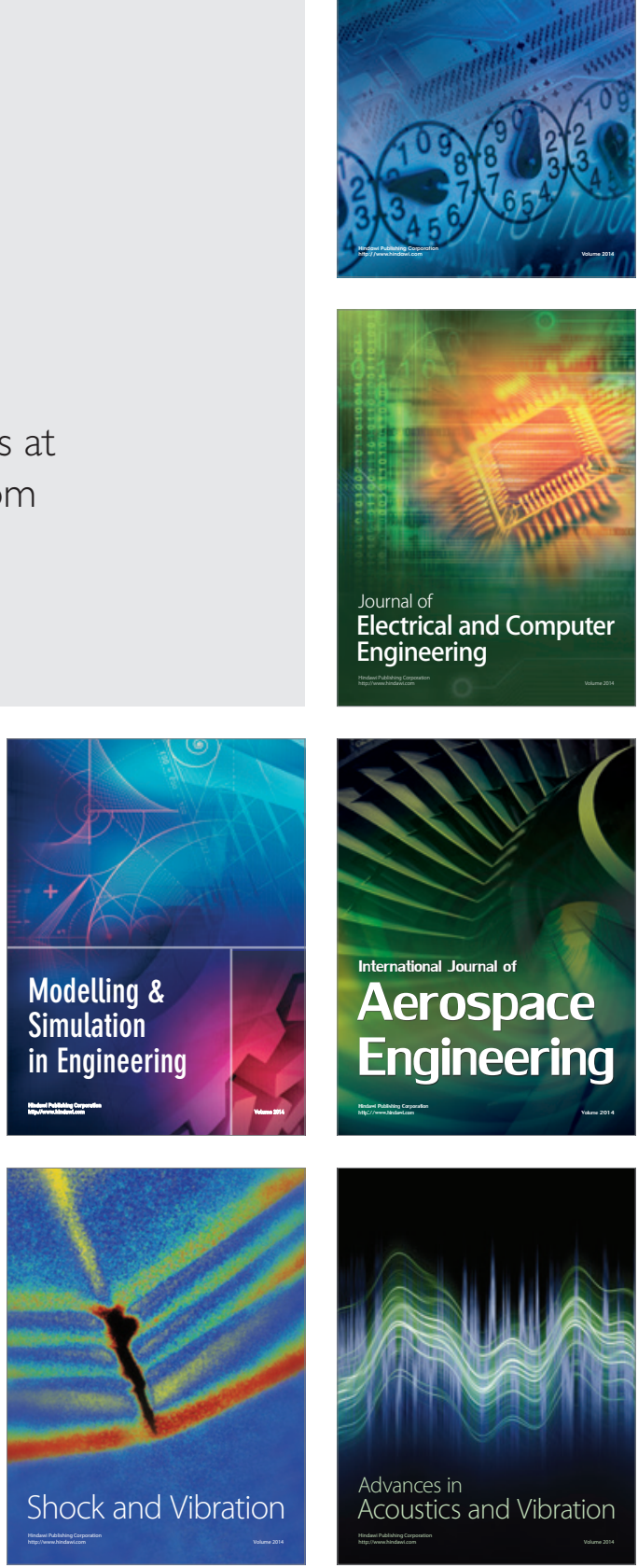